\title{
Divergence in a stress regulatory network underlies differential growth
}

\section{control}

Authors: Ying $\mathrm{Sun}^{1} \uparrow$, Dong-Ha $\mathrm{Oh}^{2} \dagger$, Lina Duan ${ }^{1}$, Prashanth Ramachandran ${ }^{1}$, Andrea

\section{Affiliations:}

$6 \quad{ }^{1}$ Department of Biology, Stanford University; Stanford, CA, USA

$7 \quad{ }^{2}$ Department of Biological Sciences, Louisiana State University; Baton Rouge, LA, USA

$8{ }^{3}$ Genomic Analysis Laboratory, Salk Institute for Biological Studies; La Jolla, CA, USA

$9 *$ Corresponding author. Email: dinneny@stanford.edu

$10 \dagger$ These authors contributed equally to this work

11 Abstract: The phytohormone abscisic acid (ABA) is a central regulator of acclimation during

12 environmental stress. While some plants exhibit tremendous stress resilience, it has been unclear

13 whether differences in ABA response underlie such adaptations. Here we establish a cross-

14 species gene regulatory network $(\mathrm{GRN})$ for $\mathrm{ABA}$ to identify broadly conserved, core

15 components of the ABA signaling network and peripheral pathways exhibiting species-specific

16 connectivity. Genes that are broadly conserved in the network share promoter architecture and

17 patterns of gene expression. Networks associated with growth hormones exhibited highly

18 divergent wiring of their ABA network leading to changes in the physiological outcome of

19 signaling. Together our study provides a model for understanding how GRN subcircuits deploy

20 different growth regulatory states across ecologically diverse species. 
21 One Sentence Summary: Comparative studies reveal core and peripheral stress-mediated gene

22 networks driving divergent growth control in plants.

\section{Main Text:}

24 The most widely characterized plants are typically sensitive to stress, thus limiting our

25 knowledge of the most effective response mechanisms that exist, and our ability to design

26 innovative engineering strategies to maintain agricultural yields in the face of climate change (1-

27 3). Extremophytes are plant species that have evolved mechanisms to cope with severe abiotic

28 stresses such as salinity, drought, and cold $(4,5)$. Within the Brassicaceae, two emerging model

29 extremophyte species exhibit greater tolerance to soil salinity than the well-characterized plant

30 Arabidopsis thaliana (A. thaliana) $(6,7)$. Eutrema salsugineum (E. salsugineum) is found from

31 the saline soils in coastal China to the subarctic regions in Canada, and survives stress through

32 effective energy use, regulation of excess electron flow, and presence of protective barriers in the

33 root (8-10), while Schreinkiella parvula (S. parvula) grows in the Irano-Turanian region, is

34 tolerant to high levels of $\mathrm{Na}^{+}, \mathrm{K}^{+}, \mathrm{Li}^{+}, \mathrm{Mg}^{2+}$, and Boron $(11,12)$, but the adaptive strategies used

35 are less-well characterized. S. parvula is believed to have evolved salinity tolerance

36 independently of E. salsugineum as the closely related species Sisymbrium irio (S. irio) together

37 with the entire clade of Brassica are also stress sensitive (Fig. 1A) (13).

We chose to focus our comparative analysis on the response to abscisic acid (ABA) as a

39 proxy for environmental stress as the transcriptional response to this hormone is not dependent

40 on the organism experiencing physiological stress, which may occur under highly different

41 environmental conditions between species (14). ABA biosynthesis is induced under salt stress

42 and typically inhibits germination, root growth, and gas exchange as a means of limiting growth

43 (15-17). ABA perception triggers a signaling cascade that leads to the phosphorylation of the 
44 bZIP transcription factor (TF), ABA-RESPONSIVE ELEMENT BINDING FACTORS

45 (AREB/ABFs) $(18,19)$. AREB/ABFs relocalize to the nucleus and activate gene expression by

46 binding to cis-regulatory elements (CREs) known as ABA-RESPONSIVE ELEMENTs (ABREs)

47 (Fig. 1B).

Our growth assay confirmed the substantial tolerance of $S$. parvula and E. salsugineum to

$49 \mathrm{NaCl}$ compared with A. thaliana and S. irio (Fig. 1C). In contrast, application of exogenous ABA

50 had strong inhibitory effects in A. thaliana, E. salsugineum and S. irio, while S. parvula

51 exhibited a striking growth promotion at all concentrations tested (Fig. 1D). We characterized

52 the developmental basis for the observed growth enhancement (Fig. 1E) and found that while the

53 meristem size for A. thaliana and S. parvula is reduced upon ABA treatment (Fig. 1F), final cell

54 length for $S$. parvula increased by $50 \%$ (from $\sim 100 \mu \mathrm{m}$ to $\sim 200 \mu \mathrm{m}$ ) while no change occurred in

55 A. thaliana (Fig. 1G-H). These data reveal that all species tested respond to ABA by differential

56 growth, but that the direction of this effect varied dramatically depending on the species and

57 developmental context.

To identify conserved and species-specific ABA responsive pathways, we performed

59 RNA-Seq on the roots and shoots of all 4 species treated with either mock or $10 \mu \mathrm{M}$ ABA for 3

60 hours and 24 hours (data S1-S3). We characterized the number of differentially expressed genes

61 (DEGs) and observed that $S$. parvula uniquely responded with a more sustained transcriptional

62 response in roots and shoots, which contrasted with A. thaliana, S. irio, and E. salsugineum

63 where the greatest number of DEGs was observed after 3 hours ABA treatment in root tissue

64 (Fig. 2A). Similar S. parvula-specific trends were also observed in the prevalence of expression

65 patterns identified after clustering (Fig. 2B). 
68 define 15,198 unambiguous 1-to-1 orthologous gene groups based on reciprocal homology (Fig.

$692 \mathrm{C}$, fig. S1, and data S1). Gene groups were then clustered based on their pattern of differential

70 expression to $\mathrm{ABA}$, which revealed that for each tissue and time point where expression was

71 measured, species-specific and stress-sensitive/extremophyte-specific patterns were most

72 prevalent while genes that respond across all species were less common (colored pink, Fig. 2D).

73 Leveraging the evolutionary relationships of our studied species, we developed a

74 phylogenetically informed profiling (PiP) approach that utilizes a correlation metric to establish

75 whether pairs of species exhibit a similar regulatory program for genes associated with distinct

76 functional groups defined by GO terms (fig. S2 and data S4). PiP revealed that the genes

77 annotated with the GO term "response to abscisic acid" are significantly correlated in all species

78 pairs. These data suggest broad conservation in regulation (activated or repressed) for canonical

79 targets of ABA signaling and that the regulatory network is derived from ancestral traits (Fig.

80 2E). In addition to conserved ABA response pathways, our novel analysis method revealed that

81 orthologous gene pairs associated with protein translation ("peptide biosynthetic process"), and

82 other related GO terms, exhibited no correlation or showed anticorrelation, between S. parvula

83 and other species (Fig. 2F, fig. S2, table S1). Protein translation is finely orchestrated with the

84 metabolic activity of cells and corresponds to the "growth" cellular state (20). This pattern

85 suggests an $S$. parvula-specific change in ABA-dependent regulation that diverged from the

86 common ancestor (Fig. 2F, fig. S3). Therefore, S. parvula may have evolved to interpret ABA

87 signaling as a proxy for environmental conditions to promote growth, rather than suppress

88 growth, contrasting to other species examined. 
To determine which classes of TFs are likely involved in generating the ABA-dependent

91 transcriptional responses observed in each species, we used Analysis of Motif Enrichment

92 (AME), and searched for over-represented TF binding motifs within the region located $1 \mathrm{~kb}$

93 upstream of the coding sequence (21). We identified motifs for AREB/ABF 1/2/3/4 and 31

94 additional TFs, all of which included a G-box like sequence similar to the previously defined cis-

95 regulatory element, ABRE. This motif was not observed for ABA-repressed DEGs which is in

96 line with previous studies reporting ABREs as recruiters of transcriptional activation (22) (fig.

97 S4, data S5). Our promoter analysis suggests that the molecular mechanism underlying ABA-

98 induced gene expression is functionally conserved across species.

Based on this evidence for a shared mechanism of ABA-dependent gene expression, we

100 sought to establish comparative global maps of AREB/ABF binding landscapes since

101 AREB/ABFs are expressed in vegetative tissues (fig. S5) and play an indispensable and inducing

102 role in ABA-mediated gene expression in A. thaliana (18, 23-27). The recent development of

103 DNA affinity purification (DAP-Seq) provided a method amendable for the study of non-model

104 organisms (28). DAP-Seq facilitates the direct measurement of TF to genomic DNA (gDNA)

105 binding without the need to develop TF-specific antibodies or generate transgenic lines with

106 epitope-tagged TFs. Despite differences in target gene expression, the AREB/ABF gene family

107 has not expanded or contracted, resulting in 4 homologs within each species (fig. S6).

108 Overall, thousands of AREB/ABF-target associations were identified: 14,374 for $A$.

109 thaliana, 10,558 for S. irio, 10,197 for S. parvula, and 20,026 for E. salsugineum. We uncovered

110 highly similar ABRE-like motifs as the most predominant binding site, which is of particular

111 significance as no prior information on TF-DNA interactions were available for $S$. irio, $S$.

112 parvula, and E. salsugineum (Fig. 3A, fig. S6-7, data S1-2 and S6). While the core ACGT 
113 sequence motif does not exhibit enrichment across gene regions, ABREs and AREB/ABF

114 binding occurs to a greater extent in the 5' promoter region (fig. S8, data S6). The top 3,000 sites

115 targeted by each AREB/ABF paralog were highly similar within each species (Fig. 3B). To

116 further investigate these relationships, we swapped the cognate genome for a common

117 noncognate genome (A. thaliana) during the pull-down, which we termed swap-DAP; again, we

118 found a large overlap of binding positions between the respective AREB/ABF paralogs to the $A$.

119 thaliana genome. Together these results suggest that AREB/ABF-DNA binding dynamics are

120 highly similar in Brassicaceae and between paralogous AREB/ABFs and that differences in the

121 coding sequence of the AREB/ABF likely play a minor role in determining differences in ABA-

122 dependent gene expression (Fig. 3B, fig. S9).

123 We next investigated whether variation in promoter sequence best explained the

124 divergence in ABA response between species. Within our dataset, we observed several

125 incidences where the ABF binding profile deviates in a species-specific manner, an example of

126 which is shown for a molecular chaperone important for stress tolerance (AT2G21510) (Fig. 3C)

127 (29). In this case, AREB/ABF binding is associated with greater transcript abundance under 10

$128 \mu \mathrm{M}$ ABA treatment for all species except $S$. parvula, suggesting that these changes are cis-

129 regulatory effects. Alignment of promoter sequences associated with the AREB/ABF binding

130 site identified a single base pair difference in the core ABRE motif of the S. parvula promoter

131 despite other ABRE-like sequences being present (Fig. 3D), suggesting that a well-positioned

132 base pair mutation may be sufficient to disrupt AREB/ABF binding and subsequent gene

133 expression.

134 To globally establish how differences in AREB/ABF binding relate to promoter sequence 135 variation, we compared the 5' sequence similarity of orthologous genes, focusing on A. thaliana 
136 and S. parvula (Fig. 3E-H). Gene pairs where both members had significant AREB/ABF binding

137 in this region were much more likely to exhibit sequence similarity across their entire 5' region

138 compared to gene pairs where neither member, or only one, had an AREB/ABF binding event

139 (Fig. 3E). This pattern of conservation may indicate that additional sequence features, such as

140 other CREs or overall promoter architecture, are needed to facilitate binding of the AREB/ABF.

141 We searched for the presence of ABRE-like sequences within the regions underlying

142 AREB/ABF-binding events deduced from DAP-Seq peaks. For orthologous gene pairs

143 exhibiting presence/absence variation (PAV) in ABF binding, the most frequent underlying

144 difference was the complete gain/loss of the sequence block containing the ABRE (Fig. 3F,

145 "Structural variation") followed by point mutations disrupting the ABRE ("CRE modified").

146 Only $21 \%$ of PAVs occurred despite a conserved CRE and homologous neighboring sequence,

147 which could be explained by variation in flanking/distal sequences or epigenetic context (30).

148 For gene pairs with conserved ABF binding, most promoter pairs had a conserved ABRE

149 sequence underlying the peak (Fig. 3G), as expected. These data highlight the potential role of

150 single nucleotide polymorphisms (SNPs) and insertions/deletions (indels) as frequent drivers of

151 divergence in the ability of promoters to recruit TFs that may lead to the acquisition of lineage

152 specific traits.

We next sought to define an AREB/ABF-centered gene regulatory network (GRN) for

154 ABA signaling that established conserved, core components of the network as well as peripheral

155 modules that exhibited species-specific divergence (31). A conserved GRN was established by

156 identifying 354 high confidence AREB/ABF targets (HCATs) that responded across species (320

157 for 3 hours root, 84 for 24 hours root, 45 for 3 hours shoot, and 47 for 24 hours shoot) and had

158 AREB/ABF binding (Fig. 4A). While AREB/ABF binding to the 5' proximal region of genes 
was best correlated with differential gene expression (fig. S10, table S2), we observed that

160 orthologous HCAT genes also showed conserved patterns of AREB/ABF binding in the gene

161 body or 3' untranslated regions (Fig. 4B, upper panel). The magnitude of ABA regulation for

162 these genes was also highly conserved across species (Fig 4B, lower panel).

We used these HCATs to construct a core conserved ABF network and identified

164 functional categories that were directly targeted by AREB/ABFs and regulated by ABA across

165 all 4 species (data S7). Curation of the genes in this network revealed that known ABA-specific

166 TFs and signaling components were well represented and tended to have multiple AREB/ABF

167 binding sites in their promoters, which correlated with a greater magnitude of ABA induction

168 across species and broad activation of expression across tissue layers in roots during salt stress,

169 based on data from A. thaliana (32) (Fig 4C). Our findings also suggest that AREB/ABFs induce

170 more nuanced tissue-specific changes in physiological responses, such as transport, which may

171 be facilitated by having fewer AREB/ABF binding sites (Fig 4C).

172 We next looked for evidence of subcircuit topologies that differed between species to

173 contextualize AREB/ABF GRN divergence in the Brassicaceae. We focused our analysis on the

174 auxin and ethylene pathways, as these hormones are known to cross-talk with ABA to mediate

175 changes in growth (33-36). In particular, we were curious to identify divergence in AREB/ABF

176 targets that would illuminate our understanding of the growth promoting response of ABA

177 observed in S. parvula. We curated a list of genes with roles in biosynthesis, transport, and

178 signaling for 199 auxin related genes and 72 ethylene related genes (fig. S11-12) (37- 47). Of

179 these genes, AREB/ABFs targeted 81 auxin related genes and 33 ethylene related genes in at

180 least one species. While we observed GRN differences in 18 out of the 33 ethylene-related

181 genes, only 2 were $S$. parvula-specific. In contrast, we observed 15 auxin-related genes with $S$. 
182 parvula-specific GRN differences (Fig. 5A). We also observed transcriptional patterns that

183 correlate with these GRN differences providing in vivo evidence for the repurposing of an auxin

184 related subnetwork in S. parvula (Fig. 5B).

Auxin inhibits root growth by suppressing the rate of cell elongation (48). Establishing an AREB/ABF-mediated GRN in $S$. parvula revealed a large number of genes that encode enzymes

187 involved in auxin biosynthesis (Fig. 5A). These include TAA1 and YUC2, which mediate the last

188 steps of auxin biosynthesis, as well as root growth inhibition in response to stress $(49,50)$. In $A$.

189 thaliana, AREB/ABF binding likely leads to the induction of these genes upon ABA treatment,

190 while in $S$. parvula, AREB/ABF binding is lost, and their expression is unaffected by ABA

191 treatment. Thus, we hypothesized that differences in the ability of ABA to inhibit growth may, in

192 part, be mediated by differential control of TAA1 or YUC2. We tested our hypothesis by using a

193 TAA1 inhibitor, L- Kyruneine, and the TAA1 mutant allele wei8-1, and found that both methods

194 of reducing auxin biosynthesis lowered the sensitivity of roots to ABA treatment in A. thaliana,

195 thus partially phenocopying what we observed in S. parvula under ABA treatment $(51,52)$.

196 These data are consistent with previous work demonstrating the role of auxin signaling in

197 mediating ABA-response (33). Finally, we found that the growth inducing effects of ABA

198 treatment on S. parvula could be reversed with supplemental auxin (Fig. 5D), thus recapitulating

199 an A. thaliana-like signaling environment. Together our findings reveal that $S$. parvula has

200 undergone extensive rewiring of its AREB/ABF network, with specific changes in the

201 connectivity to the auxin pathway and growth regulation (Fig. 5E).

\section{Conclusions}

203 We used species within the Brassicaceae to investigate how stress-mediated GRNs differed

204 between extremophyte and stress sensitive species, taking advantage of the extensive gene 
205 annotation and knowledge associated with $A$. thaliana. In building an AREB/ABF-centered

206 GRN, we identified a robust evolutionarily conserved ABA pathway, and divergence in

207 peripheral pathways that contribute to hormone cross-talk and growth regulation.

208 Previous work has proven the utility of manipulating the promoter sequences of plant

209 growth regulators to generate agriculturally beneficial traits in crops (53). The work presented

210 here has identified genes whose promoters have likely been the target of natural selection for

211 extreme stress tolerance and will likely fuel the further implementation of a genetic engineering

212 strategy for crop improvement that targets gene regulatory sequences. Future work to incorporate

213 more Brassicaceae species into a comparative framework will further elucidate the principles by

214 which divergence in GRNs contribute to novel physiological outcomes and adaptation to

215 stressful environments. 


\section{References and Notes:}

1. Y. Kazachkova, G. Eshel, P. Pantha, J. M. Cheeseman, M. Dassanayake, S. Barak, Halophytism: what have we learnt from Arabidopsis thaliana relative model systems? Plant Physiol. 178, 972-988 (2018).

2. A. Raza, A. Razzaq, S. S. Mehmood, X. Zou, X. Zhang, Y. Lv, J. Xu, Impact of climate change on crops adaptation and strategies to tackle its outcome: a review. Plants. 8 (2019), doi:10.3390/plants8020034.

3. J.-K. Zhu, The next top models. Cell (2015).

4. A. O. Shamustakimova, T. G. Leonova, V. V. Taranov, A. H. de Boer, A. V. Babakov, Cold stress increases salt tolerance of the extremophytes Eutrema salsugineum (Thellungiella salsuginea) and Eutrema (Thellungiella) botschantzevii. J. Plant Physiol. 208, 128-138 (2017).

5. A. Mishra, B. Tanna, Halophytes: potential resources for salt stress tolerance genes and promoters. Front. Plant Sci. 8, 829 (2017).

6. H.-J. Wu, Z. Zhang, J.-Y. Wang, D.-H. Oh, M. Dassanayake, B. Liu, Q. Huang, H.-X. Sun, R. Xia, Y. Wu, Y.-N. Wang, Z. Yang, Y. Liu, W. Zhang, H. Zhang, J. Chu, C. Yan, S. Fang, J. Zhang, Y. Wang, F. Zhang, G. Wang, S. Y. Lee, J. M. Cheeseman, B. Yang, B. Li, J. Min, L. Yang, J. Wang, C. Chu, S.-Y. Chen, H. J. Bohnert, J.-K. Zhu, X.-J. Wang, Q. Xie, Insights into salt tolerance from the genome of Thellungiella salsuginea. Proc. Natl. Acad. Sci. U. S. A. 109, 12219-12224 (2012).

7. M. Dassanayake, D.-H. Oh, J. S. Haas, A. Hernandez, H. Hong, S. Ali, D.-J. Yun, R. A. Bressan, J.K. Zhu, H. J. Bohnert, J. M. Cheeseman, The genome of the extremophile crucifer Thellungiella parvula. Nat. Genet. 43, 913-918 (2011).

8. W. Xuejie, Z. L. F. Shoujin, Comparative studies on growth and physiological reaction of 
Thellungiella Salsuginea under $\mathrm{NaCl}$ and $\mathrm{Na} 2 \mathrm{SO} 4$ stresses. Journal of Shandong Normal University. 1 (2007) (available at https://en.cnki.com.cn/Article_en/CJFDTotal-SDZK200701042.htm).

9. Y. P. Lee, C. Funk, A. Erban, J. Kopka, K. I. Köhl, E. Zuther, D. K. Hincha, Salt stress responses in a geographically diverse collection of Eutrema/Thellungiella spp. accessions. Funct. Plant Biol. 43, 590-606 (2016).

10. J. Wang, Q. Zhang, F. Cui, L. Hou, S. Zhao, H. Xia, J. Qiu, T. Li, Y. Zhang, X. Wang, C. Zhao, Genome-wide analysis of gene expression provides new insights into cold responses in Thellungiella salsuginea. Front. Plant Sci. 8, 713 (2017).

11. D.-H. Oh, H. Hong, S. Y. Lee, D.-J. Yun, H. J. Bohnert, M. Dassanayake, Genome structures and transcriptomes signify niche adaptation for the multiple-ion-tolerant extremophyte Schrenkiella parvula. Plant Physiol. 164, 2123-2138 (2014).

12. M. J. R. MacLeod, J. Dedrick, C. Ashton, W. W. L. Sung, M. J. Champigny, E. A. Weretilnyk, Exposure of two Eutrema salsugineum (Thellungiella salsuginea) accessions to water deficits reveals different coping strategies in response to drought. Physiol. Plant. 155, 267-280 (2015).

13. F. Orsini, M. P. D’Urzo, G. Inan, S. Serra, D.-H. Oh, M. V. Mickelbart, F. Consiglio, X. Li, J. C. Jeong, D.-J. Yun, H. J. Bohnert, R. A. Bressan, A. Maggio, A comparative study of salt tolerance parameters in 11 wild relatives of Arabidopsis thaliana. J. Exp. Bot. 61, 3787-3798 (2010).

14. H. Claeys, S. Van Landeghem, M. Dubois, K. Maleux, D. Inzé, What Is stress? Dose-response effects in commonly used in vitro stress assays. Plant Physiology. 165, 519-527 (2014).

15. S. R. Cutler, P. L. Rodriguez, R. R. Finkelstein, S. R. Abrams, Abscisic acid: emergence of a core signaling network. Annu. Rev. Plant Biol. 61, 651-679 (2010).

16. S. K. Sah, K. R. Reddy, J. Li, Abscisic acid and abiotic stress tolerance in crop plants. Front. Plant 
Sci. 7, 571 (2016).

17. K. Vishwakarma, N. Upadhyay, N. Kumar, G. Yadav, J. Singh, R. K. Mishra, V. Kumar, R. Verma, R. G. Upadhyay, M. Pandey, S. Sharma, Abscisic acid signaling and abiotic stress tolerance in plants: A review on current knowledge and future prospects. Front. Plant Sci. 8, 161 (2017).

18. T. Yoshida, Y. Fujita, K. Maruyama, J. Mogami, D. Todaka, K. Shinozaki, K. Yamaguchi $\square$ shinozaki, Four Arabidopsis AREB / ABF transcription factors function predominantly in gene expression downstream of SnRK2 kinases in abscisic acid signalling in response to osmotic stress. Plant, Cell \& Environment. 38, 35-49 (2015).

19. K. Yamaguchi-Shinozaki, K. Shinozaki, A novel cis-acting element in an Arabidopsis gene is involved in responsiveness to drought, low-temperature, or high-salt stress. Plant Cell. 6, 251-264 (1994).

20. D. Deprost, L. Yao, R. Sormani, M. Moreau, G. Leterreux, M. Nicolaï, M. Bedu, C. Robaglia, C. Meyer, The Arabidopsis TOR kinase links plant growth, yield, stress resistance and mRNA translation. EMBO Rep. 8, 864-870 (2007).

21. O. Fornes, J. A. Castro-Mondragon, A. Khan, R. van der Lee, X. Zhang, P. A. Richmond, B. P. Modi, S. Correard, M. Gheorghe, D. Baranašić, W. Santana-Garcia, G. Tan, J. Chèneby, B. Ballester, F. Parcy, A. Sandelin, B. Lenhard, W. W. Wasserman, A. Mathelier, JASPAR 2020: update of the open-access database of transcription factor binding profiles. Nucleic Acids Research (2019), doi:10.1093/nar/gkz1001.

22. Y. Fujita, M. Fujita, R. Satoh, K. Maruyama, M. M. Parvez, M. Seki, K. Hiratsu, M. Ohme-Takagi, K. Shinozaki, K. Yamaguchi-Shinozaki, AREB1 is a transcription activator of novel ABREdependent ABA signaling that enhances drought stress tolerance in Arabidopsis. Plant Cell. 17, 3470-3488 (2005). 
23. F. Li, F. Mei, Y. Zhang, S. Li, Z. Kang, H. Mao, Genome-wide analysis of the AREB/ABF gene lineage in land plants and functional analysis of TaABF3 in Arabidopsis. BMC Plant Biol. 20, 558 (2020).

24. X. Yue, G. Zhang, Z. Zhao, J. Yue, X. Pu, M. Sui, Y. Zhan, Y. Shi, Z. Wang, G. Meng, Z. Zhao, L. An, A cryophyte transcription factor, CbABF1, confers freezing, and drought tolerance in tobacco. Front. Plant Sci. 10, 699 (2019).

25. J. Liu, J. Chu, C. Ma, Y. Jiang, Y. Ma, J. Xiong, Z.-M. Cheng, Overexpression of an ABAdependent grapevine bZIP transcription factor, VvABF2, enhances osmotic stress in Arabidopsis. Plant Cell Rep. 38, 587-596 (2019).

26. S. Gao, J. Gao, X. Zhu, Y. Song, Z. Li, G. Ren, X. Zhou, B. Kuai, ABF2, ABF3, and ABF4 promote ABA-mediated chlorophyll degradation and leaf senescence by transcriptional activation of chlorophyll catabolic genes and senescence-associated genes in Arabidopsis. Mol. Plant. 9, 12721285 (2016).

27. H. Choi, J. Hong, J. Ha, J. Kang, S. Y. Kim, ABFs, a family of ABA-responsive element binding factors. J. Biol. Chem. 275, 1723-1730 (2000).

28. R. C. O’Malley, S.-S. C. Huang, L. Song, M. G. Lewsey, A. Bartlett, J. R. Nery, M. Galli, A. Gallavotti, J. R. Ecker, Cistrome and epicistrome features shape the regulatory DNA landscape. Cell. 166, 1598 (2016).

29. Y. Guo, S. Mahony, D. K. Gifford, High resolution genome wide binding event finding and motif discovery reveals transcription factor spatial binding constraints. PLoS Comput. Biol. 8, e1002638 (2012).

30. R. Wu, L. Duan, J. Pruneda-Paz, D.-H. Oh, M. P. Pound, S. A. Kay, J. R. Dinneny, The 6xABRE 
synthetic promoter enables the spatiotemporal analysis of ABA-mediated transcriptional regulation. Plant Physiol. (2018), doi:10.1104/pp.18.00401.

31. Y. Sun, J. R. Dinneny, Q\&A: How do gene regulatory networks control environmental responses in plants? BMC Biol. 16, 38 (2018).

32. J. R. Dinneny, T. A. Long, J. Y. Wang, J. W. Jung, D. Mace, S. Pointer, C. Barron, S. M. Brady, J. Schiefelbein, P. N. Benfey, Cell identity mediates the response of Arabidopsis roots to abiotic stress. Science. 320, 942-945 (2008).

33. J. M. Thole, E. R. Beisner, J. Liu, S. V. Venkova, L. C. Strader, Abscisic acid regulates root elongation through the activities of auxin and ethylene in Arabidopsis thaliana. G3: Genes, Genomes, Genetics. 4, 1259-1274 (2014).

34. W. G. Spollen, M. E. LeNoble, T. D. Samuels, N. Bernstein, R. E. Sharp, Abscisic acid accumulation maintains maize primary root elongation at low water potentials by restricting ethylene production. Plant Physiol. 122, 967-976 (2000).

35. J. H. Rowe, J. F. Topping, J. Liu, K. Lindsey, Abscisic acid regulates root growth under osmotic stress conditions via an interacting hormonal network with cytokinin, ethylene and auxin. New Phytol. 211, 225-239 (2016).

36. H. Qin, L. He, R. Huang, The coordination of ethylene and other hormones in primary root development. Front. Plant Sci. 10, 874 (2019).

37. M. Majda, S. Robert, The role of auxin in cell wall expansion. Int. J. Mol. Sci. 19 (2018), doi:10.3390/ijms19040951.

38. L. C. Strader, G. L. Chen, B. Bartel, Ethylene directs auxin to control root cell expansion. Plant J. 64, 874-884 (2010). 
39. J. Li, H.-H. Xu, W.-C. Liu, X.-W. Zhang, Y.-T. Lu, Ethylene inhibits root elongation during alkaline stress through AUXIN1 and associated changes in Auxin accumulation. Plant Physiol. 168, 1777$1791(2015)$.

40. H. Takatsuka, M. Umeda, Hormonal control of cell division and elongation along differentiation trajectories in roots. J. Exp. Bot. 65, 2633-2643 (2014).

41. J.-L. Mao, Z.-Q. Miao, Z. Wang, L.-H. Yu, X.-T. Cai, C.-B. Xiang, Arabidopsis ERF1 mediates cross-talk between ethylene and auxin biosynthesis during primary root elongation by regulating ASA1 expression. PLoS Genet. 12, e1005760 (2016).

42. L. Van den Broeck, M. Dubois, M. Vermeersch, V. Storme, M. Matsui, D. Inzé, From network to phenotype: the dynamic wiring of an Arabidopsis transcriptional network induced by osmotic stress. Mol. Syst. Biol. 13, 961 (2017).

43. Z. Yang, L. Tian, M. Latoszek-Green, D. Brown, K. Wu, Arabidopsis ERF4 is a transcriptional repressor capable of modulating ethylene and abscisic acid responses. Plant Mol. Biol. 58, 585-596 (2005).

44. M. Dubois, A. Skirycz, H. Claeys, K. Maleux, S. Dhondt, S. De Bodt, R. Vanden Bossche, L. De Milde, T. Yoshizumi, M. Matsui, D. Inzé, Ethylene Response Factor 6 acts as a central regulator of leaf growth under water-limiting conditions in Arabidopsis. Plant Physiol. 162, 319-332 (2013).

45. M. Müller, S. Munné-Bosch, Ethylene response factors: a key regulatory hub in hormone and stress signaling. Plant Physiol. 169, 32-41 (2015).

46. M. N. Markakis, T. De Cnodder, M. Lewandowski, D. Simon, A. Boron, D. Balcerowicz, T. Doubbo, L. Taconnat, J.-P. Renou, H. Höfte, J.-P. Verbelen, K. Vissenberg, Identification of genes involved in the ACC-mediated control of root cell elongation in Arabidopsis thaliana. BMC Plant 
Biol. 12, 208 (2012).

47. Z.-Q. Miao, P.-X. Zhao, J.-L. Mao, L.-H. Yu, Y. Yuan, H. Tang, Z.-B. Liu, C.-B. Xiang, HOMEOBOX PROTEIN 52 mediates the crosstalk between ethylene and auxin signaling during primary root elongation by modulating auxin transport-related gene expression. Plant Cell. 30, 2761-2778 (2018).

48. S. M. Velasquez, E. Barbez, J. Kleine-Vehn, J. M. Estevez, Auxin and cellular elongation. Plant Physiol. 170, 1206-1215 (2016).

49. G. Liu, S. Gao, H. Tian, W. Wu, H. S. Robert, Z. Ding, Local transcriptional control of YUCCA regulates auxin promoted root-growth inhibition in response to aluminium stress in Arabidopsis. PLoS Genet. 12, e1006360 (2016).

50. Q. Wang, G. Qin, M. Cao, R. Chen, Y. He, L. Yang, Z. Zeng, Y. Yu, Y. Gu, W. Xing, W. A. Tao, T. $\mathrm{Xu}$, A phosphorylation-based switch controls TAA1-mediated auxin biosynthesis in plants. Nat. Commun. 11, 679 (2020).

51. D. Pacheco-Villalobos, M. Sankar, K. Ljung, C. S. Hardtke, Disturbed local auxin homeostasis enhances cellular anisotropy and reveals alternative wiring of auxin-ethylene crosstalk in Brachypodium distachyon seminal roots. PLoS Genet. 9, e1003564 (2013).

52. A. N. Stepanova, J. Robertson-Hoyt, J. Yun, L. M. Benavente, D.-Y. Xie, K. Dolezal, A. Schlereth, G. Jürgens, J. M. Alonso, TAA1-mediated auxin biosynthesis is essential for hormone crosstalk and plant development. Cell. 133, 177-191 (2008).

53. D. Rodríguez-Leal, Z. H. Lemmon, J. Man, M. E. Bartlett, Z. B. Lippman, Engineering quantitative trait variation for crop improvement by genome editing. Cell. 171, 470-480.e8 (2017).

54. J. Schindelin, I. Arganda-Carreras, E. Frise, V. Kaynig, M. Longair, T. Pietzsch, S. Preibisch, C. 
Rueden, S. Saalfeld, B. Schmid, J.-Y. Tinevez, D. J. White, V. Hartenstein, K. Eliceiri, P. Tomancak, A. Cardona, Fiji: an open-source platform for biological-image analysis. Nature Methods. 9, 676-682 (2012).

55. B. Patel, Simple, fast, and efficient cloning of PCR products with TOPO® cloning vectors. BioTechniques. 46, 559 (2009).

56. A. Haudry, A. E. Platts, E. Vello, D. R. Hoen, M. Leclercq, R. J. Williamson, E. Forczek, Z. JolyLopez, J. G. Steffen, K. M. Hazzouri, K. Dewar, J. R. Stinchcombe, D. J. Schoen, X. Wang, J. Schmutz, C. D. Town, P. P. Edger, J. C. Pires, K. S. Schumaker, D. E. Jarvis, T. Mandáková, M. A. Lysak, E. van den Bergh, M. E. Schranz, P. M. Harrison, A. M. Moses, T. E. Bureau, S. I. Wright, M. Blanchette, An atlas of over 90,000 conserved noncoding sequences provides insight into crucifer regulatory regions. Nat. Genet. 45, 891-898 (2013).

57. R. M. Waterhouse, M. Seppey, F. A. Simão, M. Manni, P. Ioannidis, G. Klioutchnikov, E. V. Kriventseva, E. M. Zdobnov, BUSCO applications from quality assessments to gene prediction and phylogenomics. Mol. Biol. Evol. 35, 543-548 (2018).

58. M. Pertea, D. Kim, G. M. Pertea, J. T. Leek, S. L. Salzberg, Transcript-level expression analysis of RNA-seq experiments with HISAT, StringTie and Ballgown. Nat. Protoc. 11, 1650-1667 (2016).

59. M. I. Love, W. Huber, S. Anders, Moderated estimation of fold change and dispersion for RNA-seq data with DESeq2. Genome Biol. 15, 550 (2014).

60. D.-H. Oh, M. Dassanayake, Landscape of gene transposition-duplication within the Brassicaceae family. DNA Res. 26, 21-36 (2019).

61. M. Steinegger, J. Söding, MMseqs2 enables sensitive protein sequence searching for the analysis of massive data sets. Nat. Biotechnol. 35, 1026-1028 (2017). 
62. S. Maere, K. Heymans, M. Kuiper, BiNGO: a Cytoscape plugin to assess overrepresentation of gene ontology categories in biological networks. Bioinformatics. 21, 3448-3449 (2005).

63. G. Wang, D.-H. Oh, M. Dassanayake, GOMCL: a toolkit to cluster, evaluate, and extract nonredundant associations of Gene Ontology-based functions. BMC Bioinformatics. 21, 139 (2020).

64. R. C. McLeay, T. L. Bailey, Motif Enrichment Analysis: a unified framework and an evaluation on ChIP data. BMC Bioinformatics. 11, 165 (2010).

65. P. Virtanen, R. Gommers, T. E. Oliphant, M. Haberland, T. Reddy, D. Cournapeau, E. Burovski, P. Peterson, W. Weckesser, J. Bright, S. J. van der Walt, M. Brett, J. Wilson, K. J. Millman, N. Mayorov, A. R. J. Nelson, E. Jones, R. Kern, E. Larson, C. J. Carey, İ. Polat, Y. Feng, E. W. Moore, J. VanderPlas, D. Laxalde, J. Perktold, R. Cimrman, I. Henriksen, E. A. Quintero, C. R. Harris, A. M. Archibald, A. H. Ribeiro, F. Pedregosa, P. van Mulbregt, SciPy 1.0 Contributors, SciPy 1.0: fundamental algorithms for scientific computing in Python. Nature Methods. 17, 261-272 (2020).

66. D. M. Emms, S. Kelly, OrthoFinder: solving fundamental biases in whole genome comparisons dramatically improves orthogroup inference accuracy. Genome Biol. 16, 157 (2015).

67. B. Langmead, S. L. Salzberg, Fast gapped-read alignment with Bowtie 2. Nature Methods (2012).

68. A. R. Quinlan, I. M. Hall, BEDTools: a flexible suite of utilities for comparing genomic features. Bioinformatics. 26, 841-842 (2010).

69. W. J. R. Longabaugh, BioTapestry: a tool to visualize the dynamic properties of gene regulatory networks. Methods Mol. Biol. 786, 359-394 (2012).

Acknowledgments: We would like to acknowledge Mary Galli and Muh Ching Yee for advice on experimental design, Carnegie Institution for Science, Department of Plant Biology and Garret Huntress for providing access to computing resources and data management, 
undergraduates Rachel Gates and Jen Pulido for their summer engagements with this study, and Chris Pires for S. irio seeds.

\section{Funding:}

US Department of Energy's Biological and Environmental Research program (Grant DESC0020358, to J.R.D., D.H.O and M.D)

Carnegie Institution for Science endowment (J.R.D)

National Science Foundation (MCB-1616827 and NSF-IOS-EDGE-1923589) (DO and MD)

RDA, South Korea (Next-Generation BioGreen21 program PJ01317301) (DO and MD)

National Science Foundation Graduate Research Fellowship (Y.S.)

HHMI-Simons Faculty Scholar (J.R.D)

\section{Author contributions:}

YS and LD performed the experiments.

YS, DO, PR, AR and JRD analyzed the data.

YS and JRD wrote the manuscript.

DO, LD, and PR contributed to the manuscript preparation.

Competing interests: Authors declare no competing interests.

Data and materials availability: All data are available in the manuscript, in the supplementary material, or in the following databases: high-throughput sequencing data sets are available through the National Center for Biotechnology Information Sequence Read Archive (NCBI SRA) under BioProject ID PRJNA682697 (https://www.ncbi.nlm.nih.gov/bioproject/682697). 
The custom scripts used to analyze the data, along with step-by-step procedures, are available at https://github.com/dinnenylab/BrassicaceaeGRN. Supplementary data are available on FigShare at https://figshare.com/s/988a4f4996a97f7824a1 (DOI:10.6084/m9.figshare.14033822). Genome browser view of data can be found on Jbrowse: http://jbrowsedap.s3-website-us-west1.amazonaws.com.

\section{Supplementary Materials:}

Materials and Methods

Figures 1-5

References (01-69)

\section{Fig. 1. Comparative analysis of stress response in the Brassicaceae family. (A) A}

phylogenetic tree generated with lineage I, stress sensitive A. thaliana, lineage II stress sensitive S. irio and extremophytes $S$. parvula and E. salsugineum. Scale = amino acid per site. (B)

Environmental stress triggers a signaling cascade and subsequent phosphorylation of bZIP TFs AREB/ABFs, which activates gene expression by binding to ABA-RESPONSIVE ELEMENTs (ABREs) (C,D) Quantification of $A$. thaliana and S. parvula primary roots after transfer to $\mathrm{NaCl}$ (C), or ABA (D) media for 2 days. Asterisks mark significant differences from A. thaliana based on two-way ANOVA, $* P<0.05, * * P<0.01, * * * P<0.001, \mathrm{n}>6$. (E) Confocal images of primary roots grown on $10 \mu \mathrm{M}$ ABA or standard media. Blue bars label the stabilized cortex cell length. Red arrows point to the end of the meristem, based on the root cap landmark. Scale bars $=100 \mu \mathrm{m}$. (F) Quantifications of root meristem size for A. thaliana and S. parvula under control and 1 or $10 \mu \mathrm{M}$ ABA treatment. Pairwise T-test, $* * * P<0.001, \mathrm{n}=5$. $(\mathbf{G}, \mathbf{H})$ Quantification of 
cortex cell length $(n=5)$. The x-axis indicates cell number starting from the quiescent center (QC).

\section{Fig. 2. RNA-Seq identifies conserved and species-specific ABA responsive pathways. (A)}

Number of differentially expressed genes (DEGs) based on $P<0.05$. (B) Heatmap of $\log _{2}$ fold changes (LFC) for DEG clusters. (C) Number of 1:1 orthologous groups (OGs) identified for any two, three, or all four species. Error bars indicate standard deviations. (D) Top 9 categories of DEGs from 1:1 OGs in descending order. The conserved responses (pink) were found to be less prevalent while responses specific to stress-sensitive plants (light and dark blue) and extremophytes (yellow and red) were most prevalent. (E,F) Phylogenetically-informed profiling (Pip) identified functional categories with lineage-specific modifications (fig. S2). Spearman's correlations $\left(\rho, * P a d j<10^{-7}\right)$ and linear regressions (with gray-shaded 95\% confidence intervals) were shown for LFC of ortholog pairs annotated with the GO term. Red arrows mark modified lineages. "ABA-response" was conserved in all species while "peptide biosynthetic process" showed S. parvula-specific deviation from conserved gene expression (gold arrows). S. parvulaspecific modifications represented the most frequent lineage-specific pattern (fig. S3a). "Peptide biosynthetic process" and related GO terms in root samples showed the most dramatic variance across species among tested GO terms (fig. S3b, table S1, and data S4).

Fig. 3. Global ABRE/ABF binding landscape demonstrates dependency between promoter sequence and TF interaction.

(A) Phylogenetic association of the amino acid sequences of 16 AREB/ABF orthologs and their most enriched motifs. Scale $=$ substitutions/site. (B) Venn Diagram showing common peak 
positions bound by AREB/ABF 1/2/3/4 for top 3000 DAP-Seq peaks. Lower panel shows top 1000 swap-DAP peaks on the A. thaliana genome. (C) Normalized genome browser window of a locus where AREB/ABF binding is absent exclusively in $S$. parvula, and correlate with gene expression differences. The y-axis for $S$. parvula was adjusted from $10,000 \mathrm{bp}$ to $50 \mathrm{bp}$ scale confirming the absence of peaks. (D) A sequence alignment of the 5' promoters of genes from (C). Blue boxes highlight ABRE motifs. Red arrow indicates the nucleotide change that leads to the loss of the core ABRE motif. (E-G) Promoter similarity distribution and sequence variations were compared for 5' $1 \mathrm{~Kb}$ promoters of $A$. thaliana and $S$. parvula ortholog pairs that showed conservation or presence/absence variation (PAV) in AREB/ABF binding. Numbers of promoter pairs were shown for each category. (H) Mode of sequence variations affecting $\mathrm{AREB} / \mathrm{ABF}$ binding (see text S1).

\section{Fig. 4. A high confidence ABRE/ABF regulatory network in Brassicaceae.}

(A) Top: Venn diagram of a shared AREB/ABF regulatory module for 1:1 ortholog groups where all orthologs are associated with a DAP-Seq peak $(2,898)$. Bottom: Venn diagram of shared AREB/ABF target genes where all orthologs were differentially expressed (3 hours roots (R03h), 24 hours roots (R24h), 3 hours shoots (S03h), 24 hours shoots (S24h), (Padj<0.05). These genes were considered as high confidence ABF targets (HCATs). (B) Top: Correlation plot comparing the number of $\mathrm{AREB} / \mathrm{ABF}$ binding sites (peaks) at different genomic features of HCATs, in A. thaliana compared with other species. Bottom: Correlation plot showing the conservation of ABA response, $\log _{2}$ fold change (LFC), in HCATs, circles indicate significant correlations calculated by Spearman's correlation $(\rho$, Padj<0.05). (C) A bubble diagram illustrating the relationship between average LFC (x-axis) and the average number of peaks 
associated with an ortholog group (y-axis) for HCATs. Bubble size indicates the number of genes, bubble color indicates average $\mathrm{NaCl}$-responsive cell types. “ABA response” genes correlate with greater number of peaks, a broader activation domain upon salt stress, and larger LFC response, while "transporter" showed fewer number of peaks, narrower expression domain, and lower LFC response.

\section{Fig. 5. Rewiring of the AREB/ABF-auxin network is responsible for altered growth} response in $S$. parvula.

(A) Divergent GRN for auxin biosynthesis, transport, signaling, and cell wall regulation are highlighted in this network composed based on literature curation. S. parvula data was overlaid onto the A. thaliana network. Species-specific differences are highlighted as well as the gain/loss of a peak based on AREB/ABF binding. (B) A heatmap summarizing the RNA-Seq data across A. thaliana and S. parvula for species-specific and lineage-specific ABA responses highlighted in the GRN. (C) Quantification of primary root growth for wild-type (WT) and wei8-1 mutants treated with mock, $1 \mu \mathrm{M}$ ABA, as well as WT with $1 \mu \mathrm{M}$ L-kynurenine (L-Kyn), and $1 \mu \mathrm{M} \mathrm{L}$ Kyn $+1 \mu \mathrm{M}$ ABA. Asterisk indicates significance by 2-way ANOVA. $* * * P<0.001, \mathrm{n}>30$. (D) Quantification of primary root growth for $S$. parvula treated with control, $1 \mu \mathrm{M}$ ABA, as well as $0.5 \mu \mathrm{M}$ IAA, $1 \mu \mathrm{M}$ IAA and co-treatment of IAA and ABA. Asterisk indicates significance by 2way ANOVA. $* * * P<0.001, \mathrm{n}>25$. (E) A schematic representation of the mechanism we proposed for the differential regulation in primary root growth in S. parvula compared with A. thaliana. 
Fig.1

A

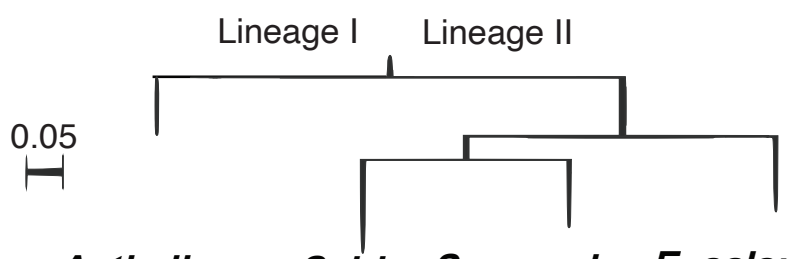

A. thaliana S. irio S. parvula E. salsugineum

Genome size $135 \mathrm{Mb}$

Chromosome 5

CDS

27,455
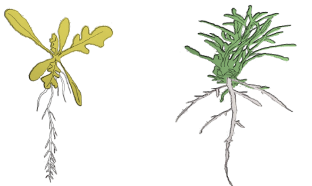

C

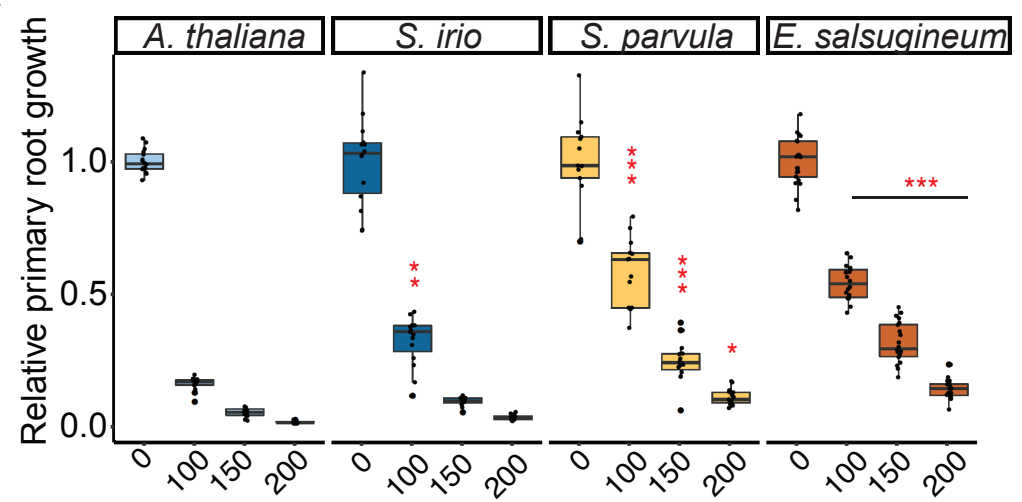

D

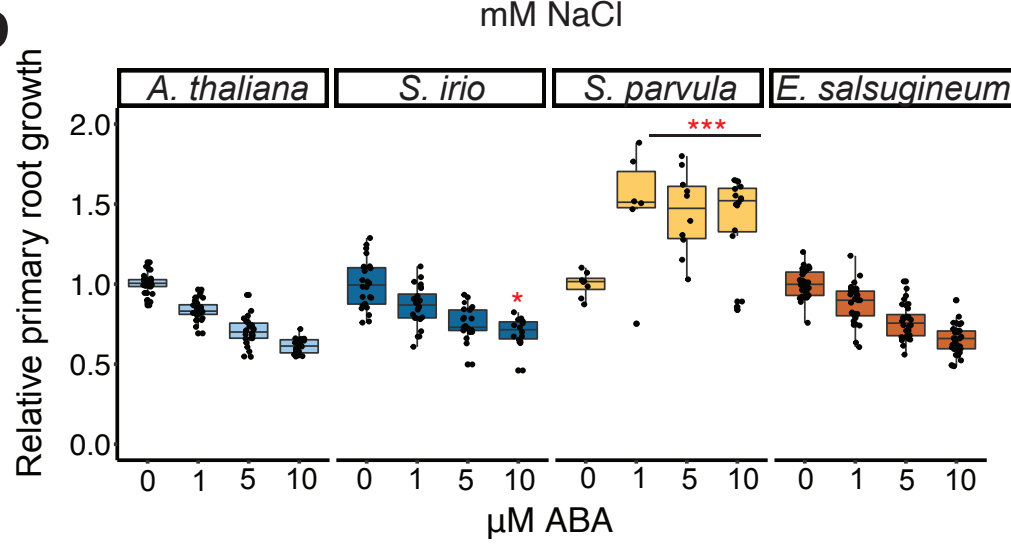

B

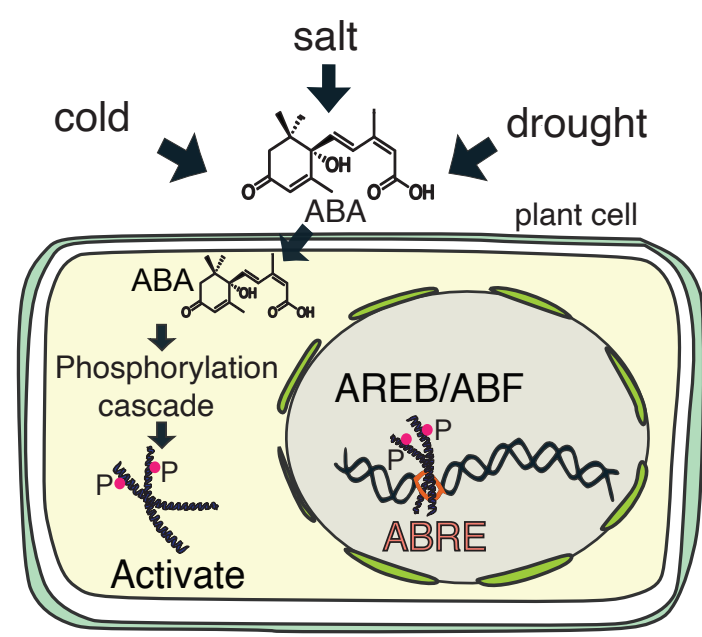

E
A. thaliana

Control $10 \mu \mathrm{M}$ ABA
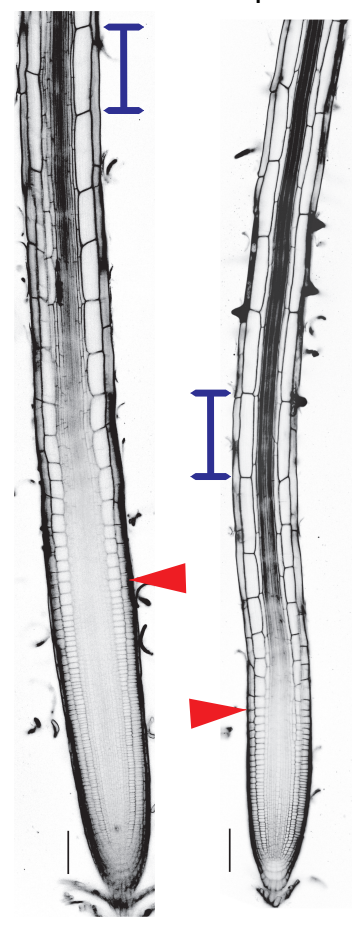

S. parvula

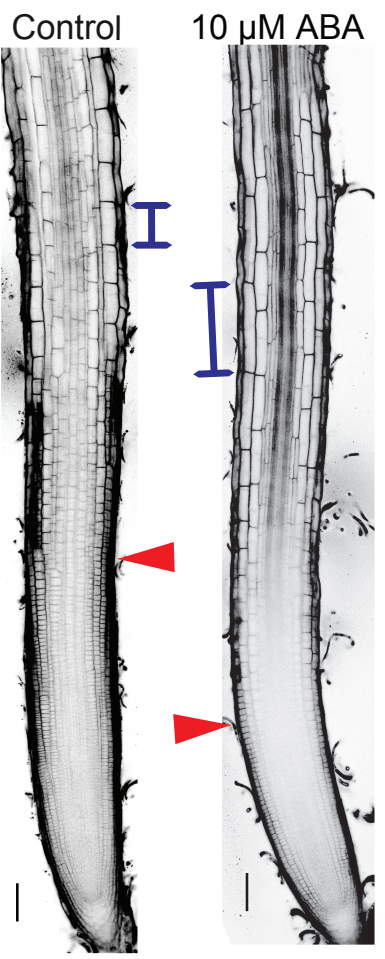

$\mathbf{F}$

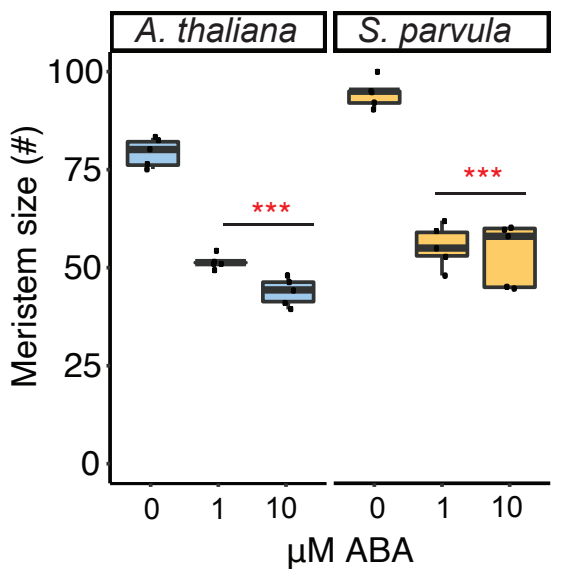

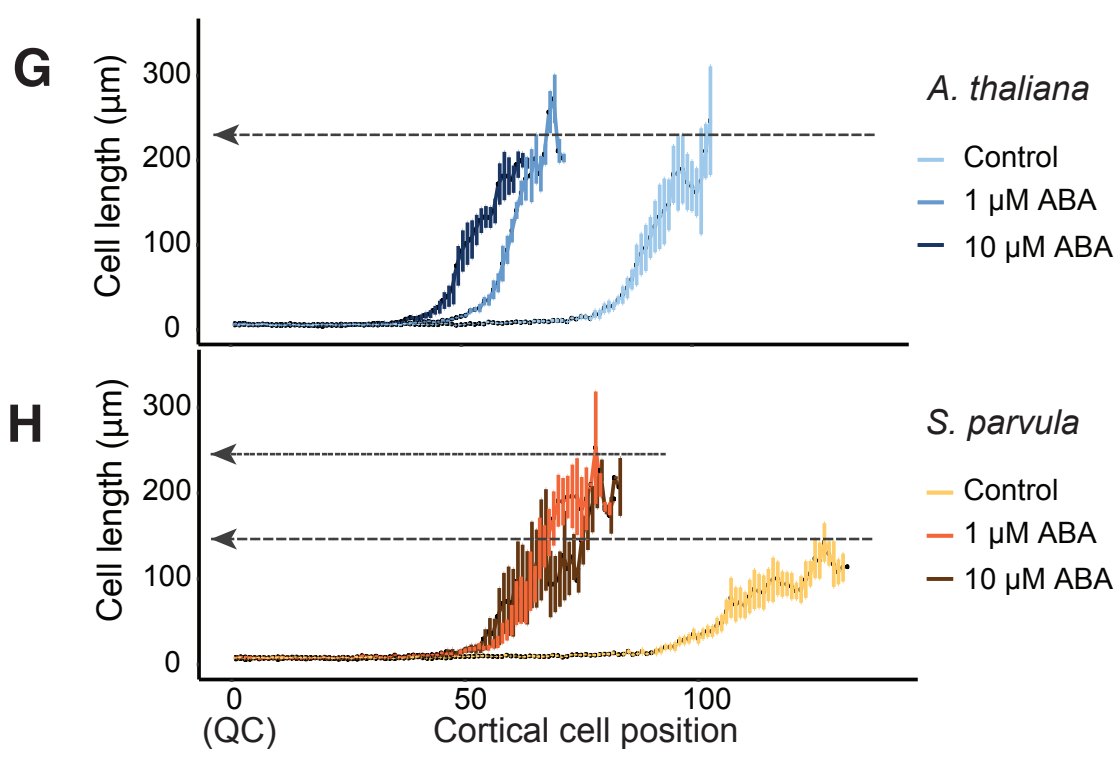


Fig. 2

A

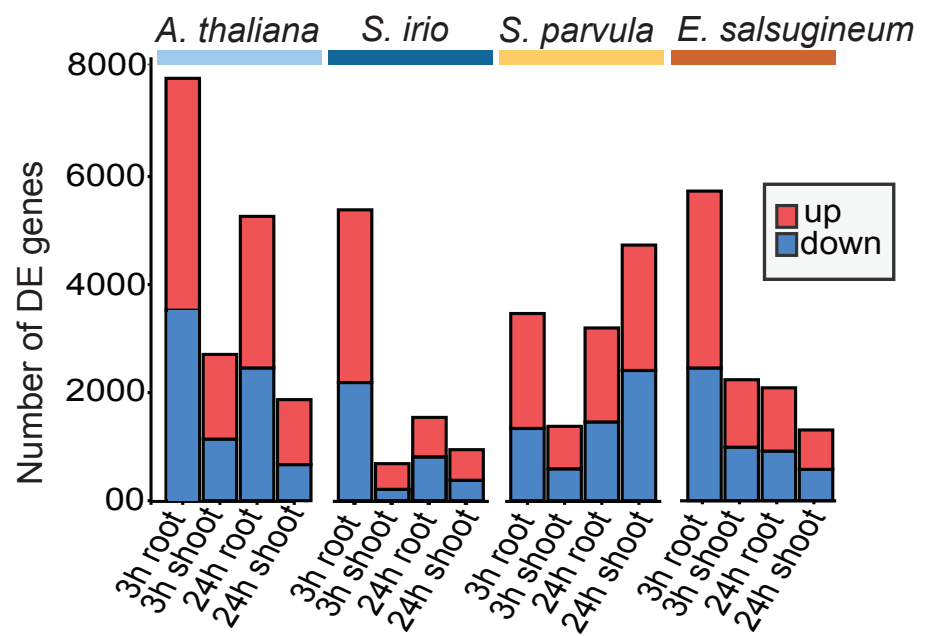

C

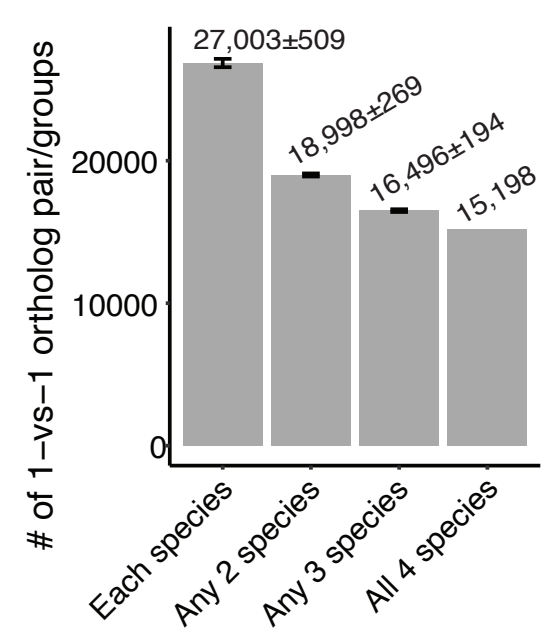

D 3 h root

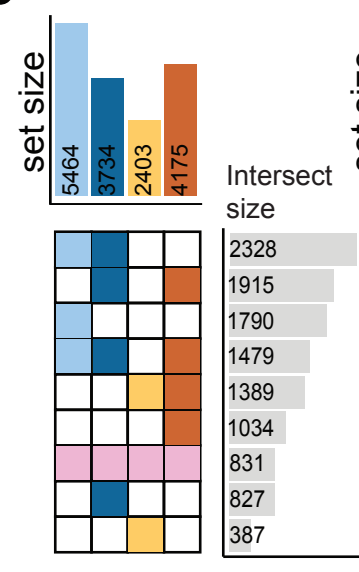

B

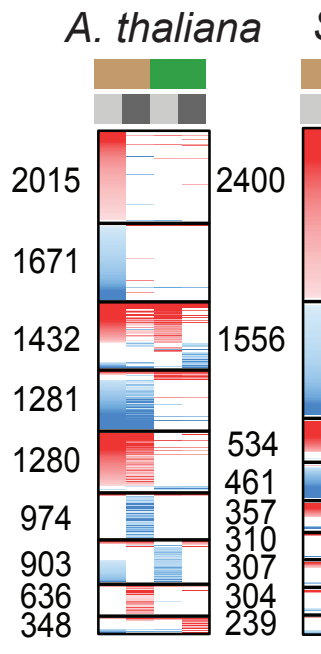

S. irio

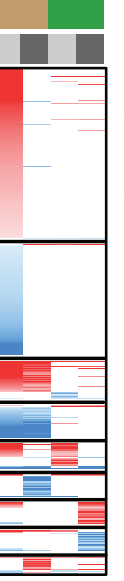

S. parvula

E. salsugineum

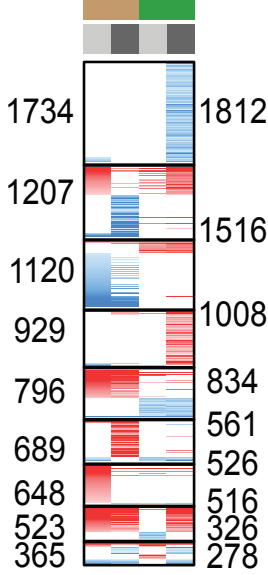

E

GO: response to abscisic acid

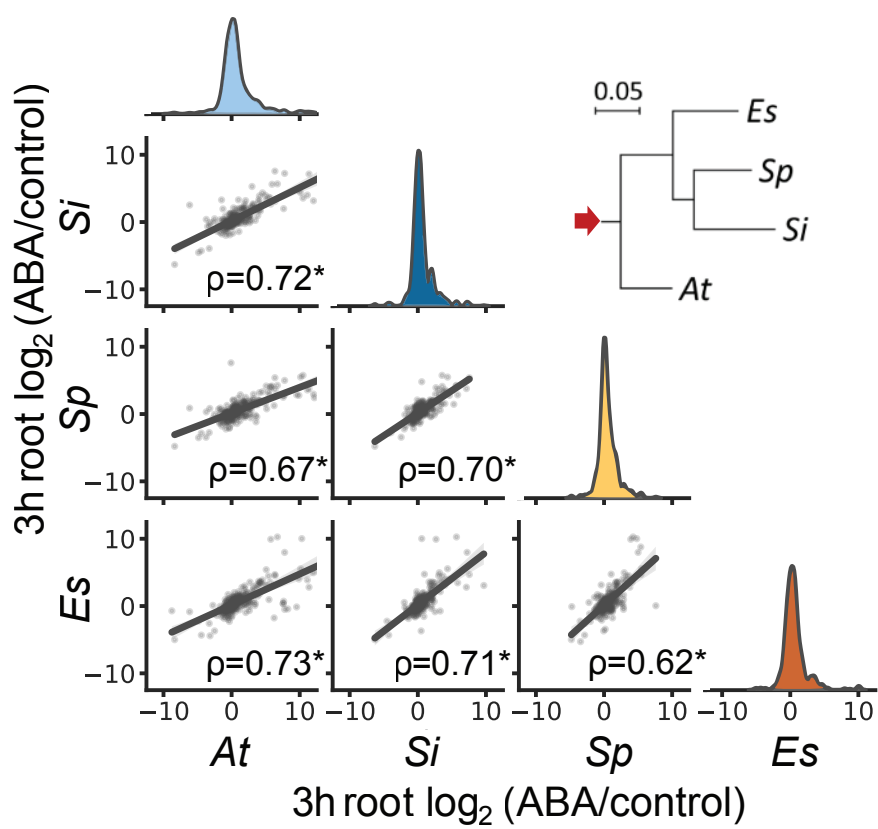

$24 \mathrm{~h}$ root

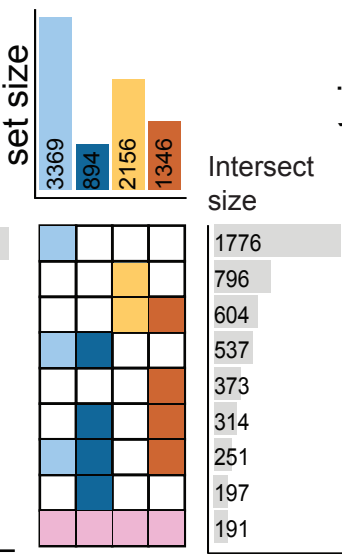

3h shoot
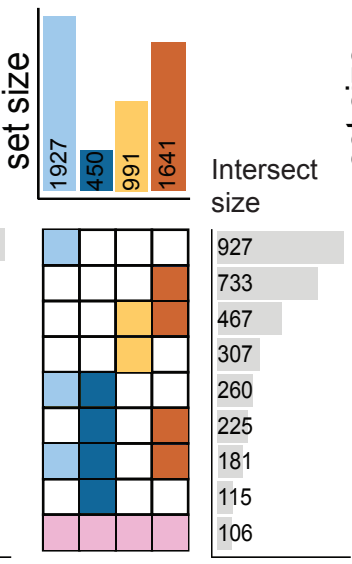

GO: peptide biosynthetic process

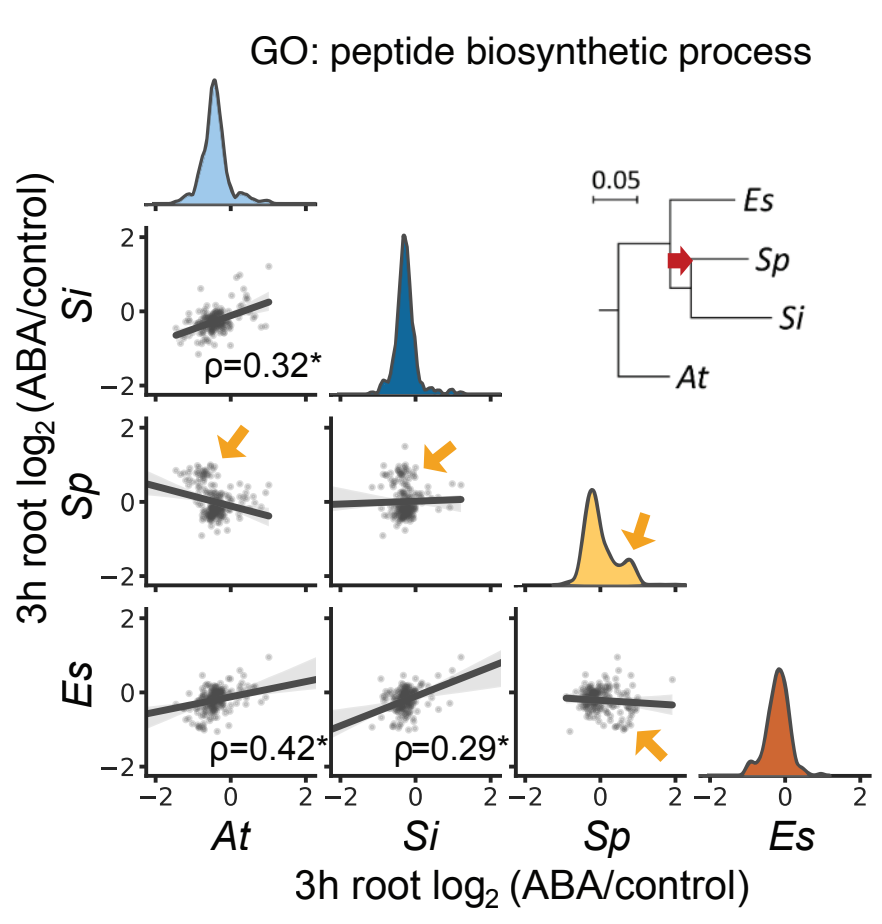

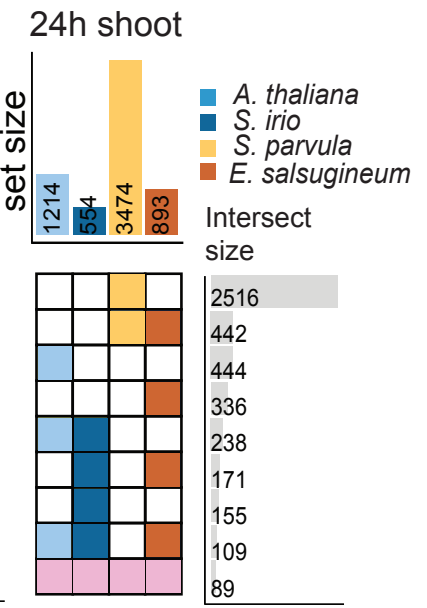

$\mathbf{F}$ 


\section{Fig. 3}

A

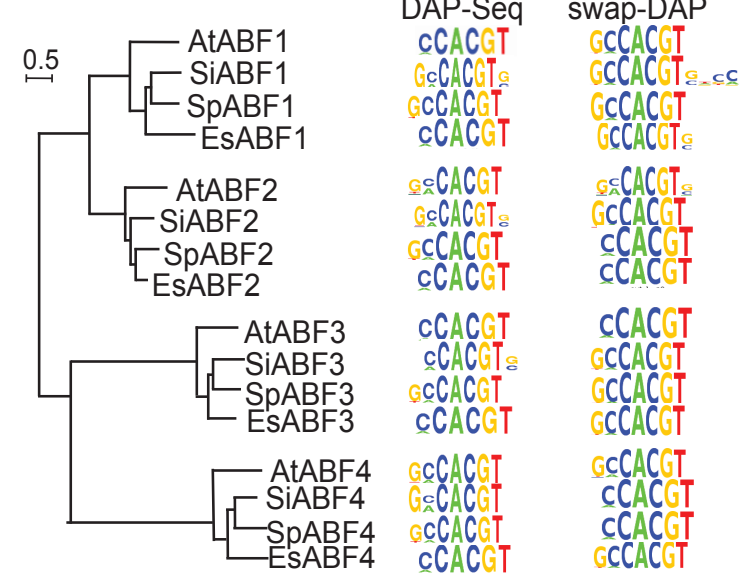

C

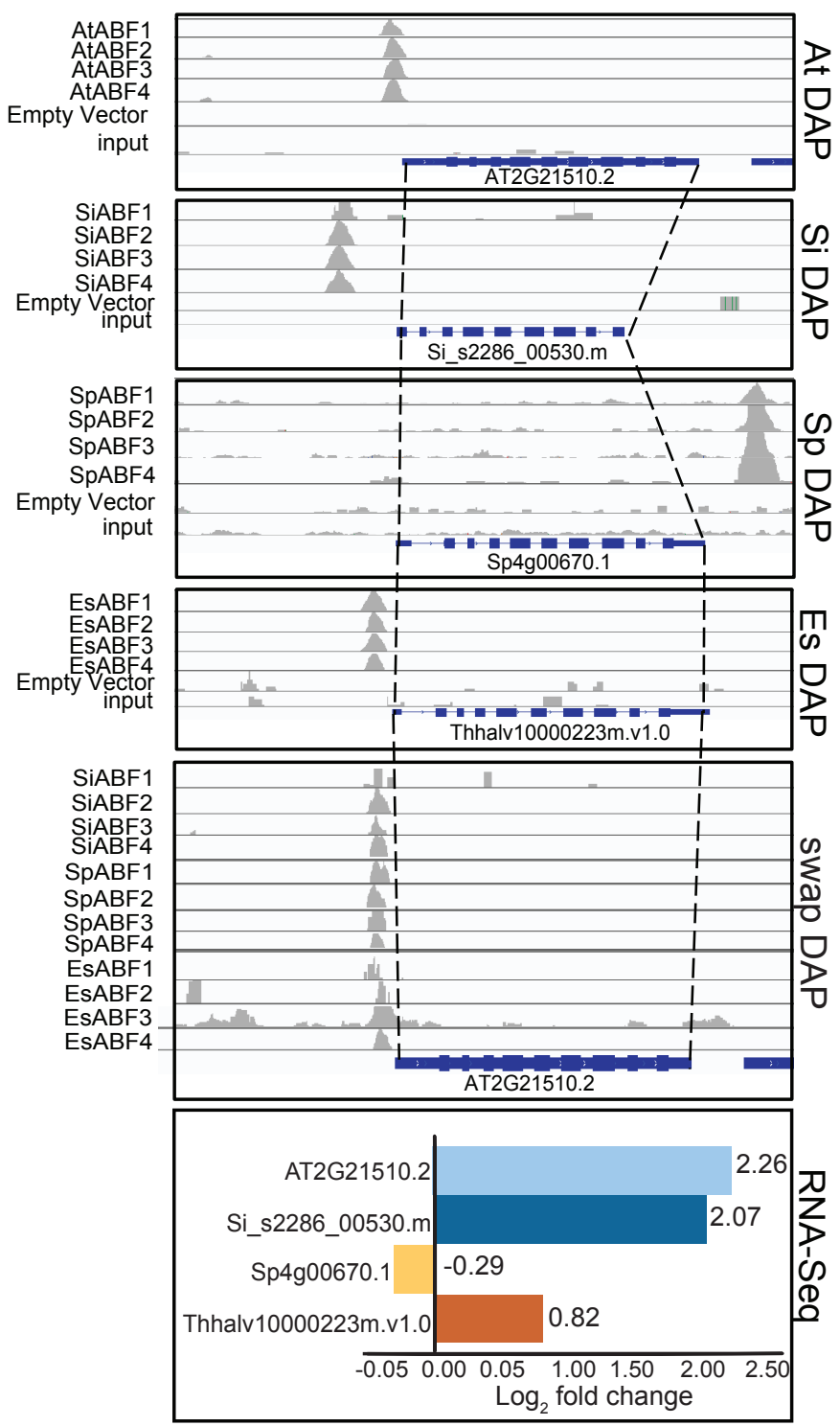

B

ABF2 ABF3 ABF2 ABF3 ABF2 ABF3 ABF2 ABF3 $\begin{array}{lllllllll}\text { ABF1 } 136 & 186 & \text { ABF4 ABF1 } 214 & 367 & \text { ABF4 ABF1 } 118 & 148 & \text { ABF4 ABF1 } 327 & 165 & \text { ABF4 }\end{array}$
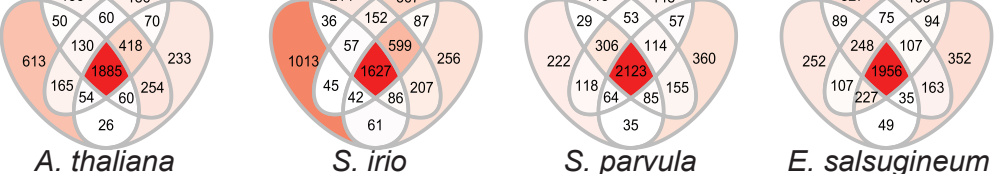

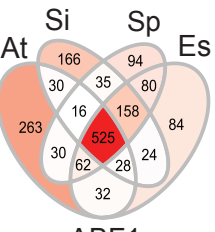

$\mathrm{ABF} 1$

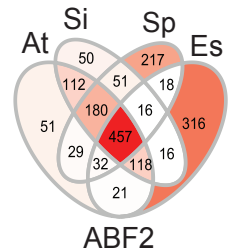

ABF2

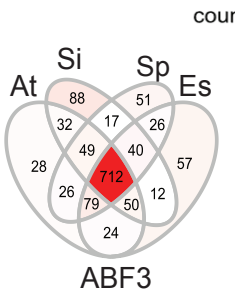

100200300400500

ABF4
D

Si CGGTTACGAAGATTATTCGA ACGTGTGACCGTGTCCGTGTTCGAATCGT--------At TG---ATAGAGA-TAGTCGAA ACGTGTGACCGTGTCCGTG------ACGTACACATCTCT

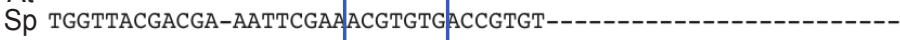

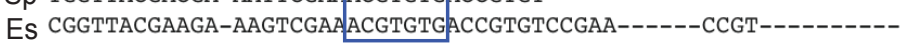

$$
\text { * } \quad * \quad * * * * * * * * * * * * * * * * * * *
$$

Si ------------- ACACGTGTCCGAGAGAATGTTCCCACTTAACCGTGTTTTGGGTTT At CTATATAATTAATACACACGTGTCC--GAGAATGTTACGACTCAACCGTGTT--.---.Sp ------------ACATGTGTCI--GAGAATGTTCTCACTGAGCCGCGTTTTTACTTES -------- ACACGTGTCC--GAGAATCTTCCTACTGAACCGTGTTTTTGGTT-
E

H

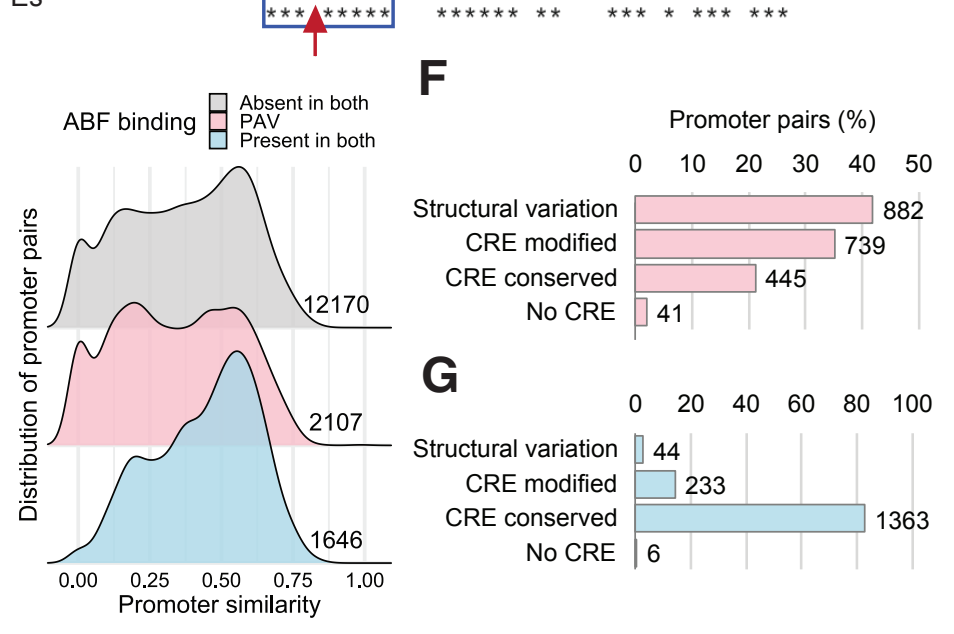

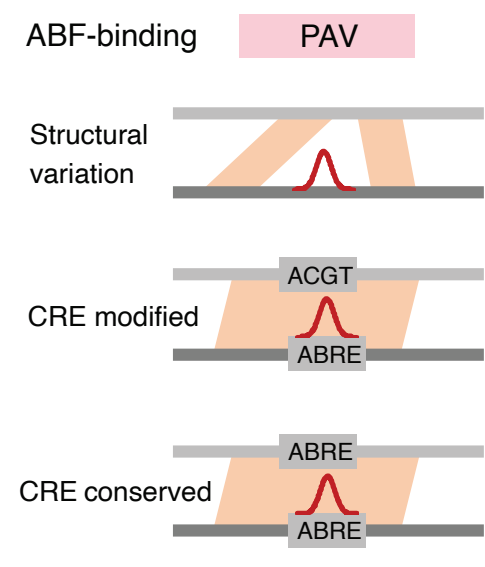

$\mathbf{F}$

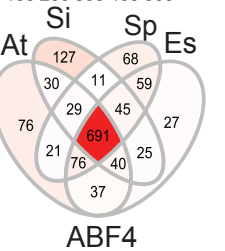

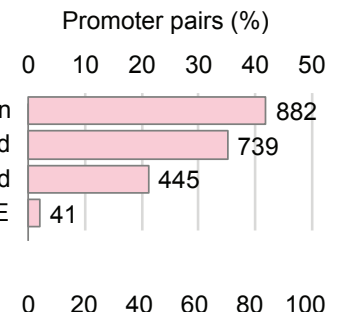

Present in both

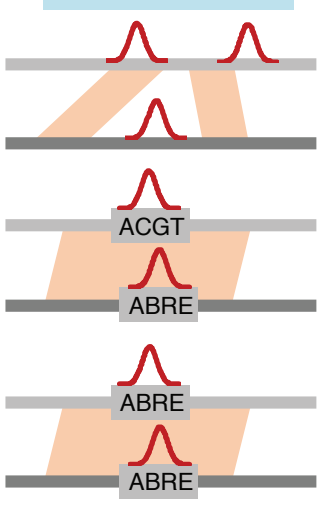
$\begin{aligned} & \text { Homologous } \\ & \text { sequence blocks }\end{aligned}=\begin{aligned} & \text { Promoters of } \\ & \text { ortholog pairs }\end{aligned} \bigwedge \begin{aligned} & \text { AREB/ABF-binding } \\ & \text { DAP-seq peak }\end{aligned}$ 
Fig. 4
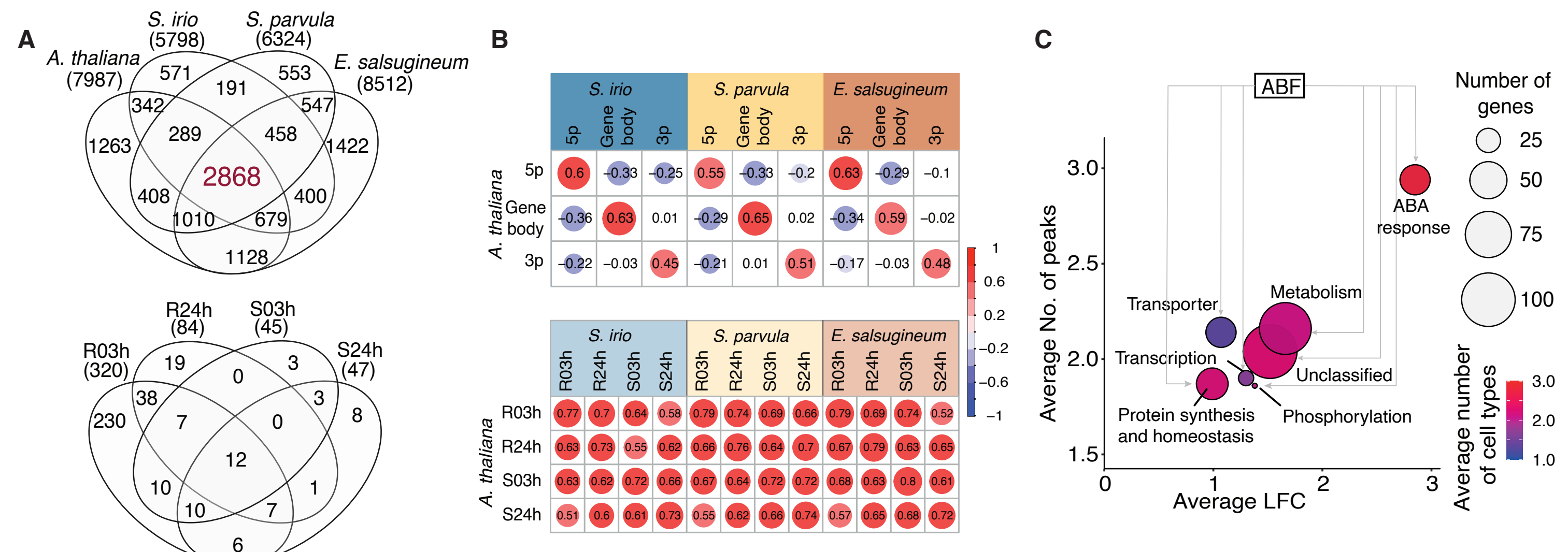
Fig. 5

A

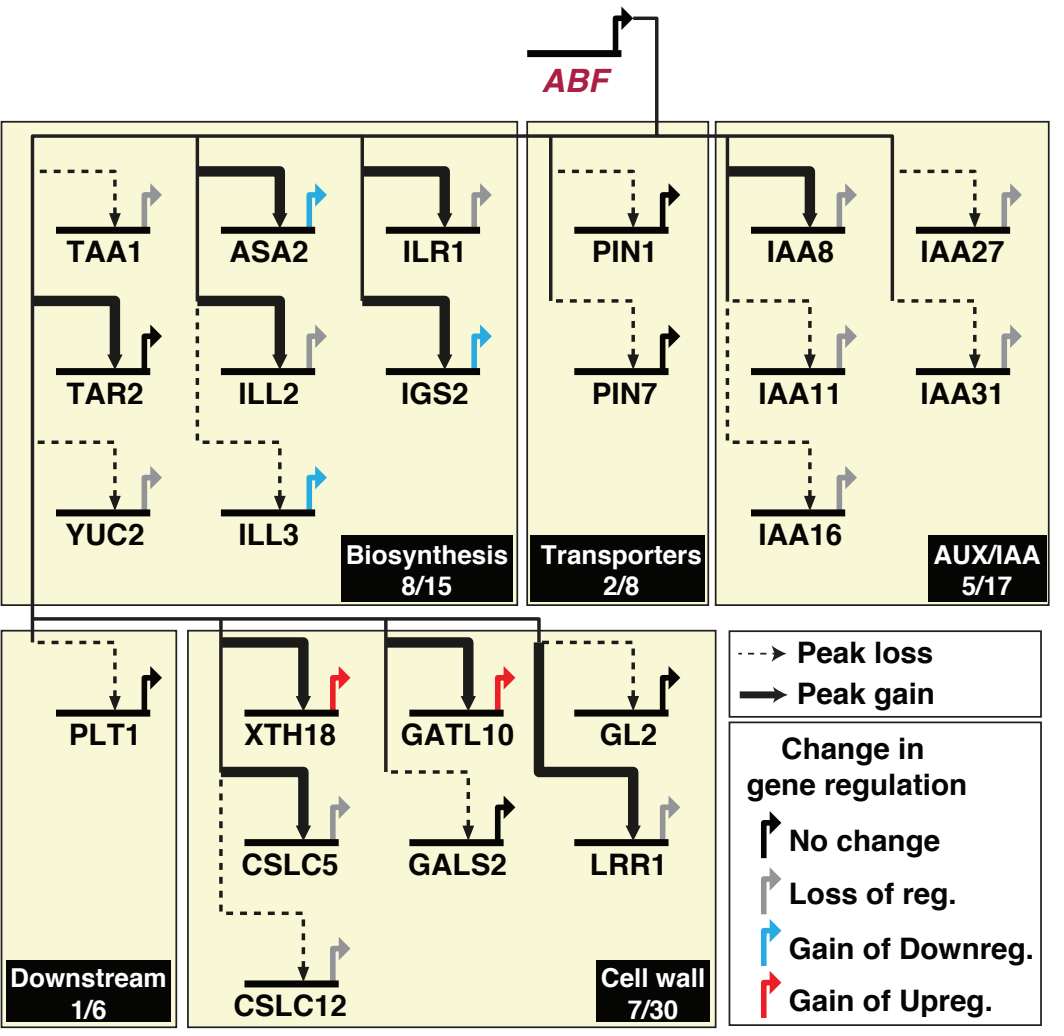

B

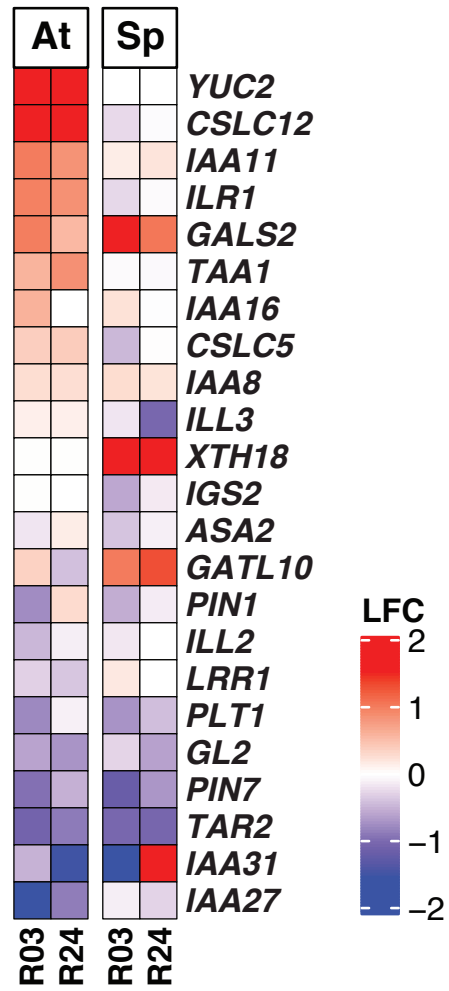

E

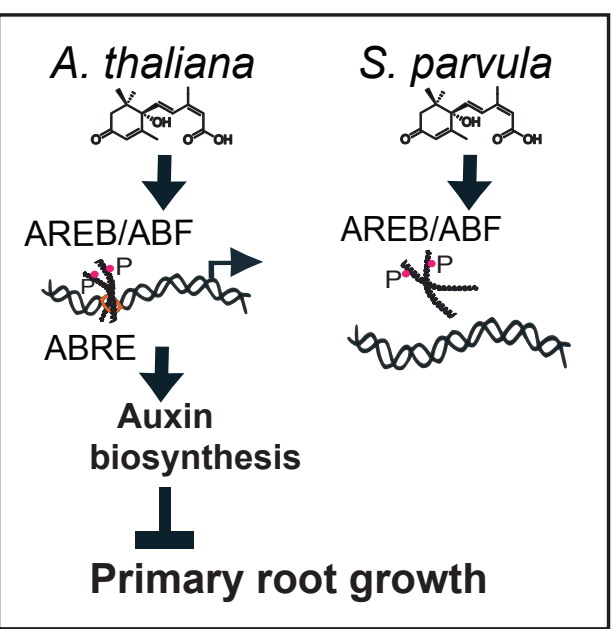

